\title{
Low grade endometrial stromal sarcoma - A series of 4 cases and review of relevant literature
}

\author{
Vijayan $\mathrm{P}^{1}$, Ilias LM ${ }^{1}$, Ponniah A ${ }^{1}$, Mohammed BA ${ }^{1}$
}

${ }^{\text {I} D e p a r t m e n t ~ o f ~ P a t h o l o g y, ~ M . E . S ~ M e d i c a l ~ C o l l e g e, ~ P e r i n t h a l m a n n a, ~ K e r a l a, ~ I n d i a ~}$

\section{Keywords: \\ Endometrial stromal \\ Sarcoma; \\ Low grade; \\ Cellular leiomyoma;}

\begin{abstract}
Endometrial stromal tumors are rare uterine malignancies composed of cells resembling those of proliferative endometrial stroma. Because of their indistinct clinical and radiological features, these malignancies are very rarely diagnosed preoperatively. They are mostly often diagnosed as fibroid uteruspreoperatively and as a result managed conservatively many a time. Usually distinguishing these tumors from benign smooth muscle tumors of the uterus are not problematic by light microscopy, however, difficulties arise in distinction between highly cellular leiomyomas and endometrial stromal sarcomas with smooth muscle differentiation. Here we present four consecutive cases of low grade endometrial stromal sarcomas with emphasis on their clinical, histopathological and immunohistochemical features. By this study, we have attempted to document the histologic features of Low grade endometrial stromal sarcoma with discussion of the differential diagnoses to contribute to this rare spectrum of uterine neoplasms.
\end{abstract}

\section{INTRODUCTION}

Mesenchymal tumors of the uterus with cytological and architectural features reminiscent of endometrial stromal cells are classified as endometrial stromal tumors (EST). ${ }^{1}$ The recent World Health Organization Classification of Tumors of the Breast and Female Genital Organs divides the uterine stromal neoplasms into three groups: (i) benign endometrial stromal nodule (ESN); (ii) low-grade endometrial stromal sarcoma (LGESS); (iii) undifferentiated endometrial sarcoma. ${ }^{2}$

Among the ESTs, Endometrial stromal sarcoma (ESS) is the commonest, accounting for $0.2-1 \%$ of all uterine malignancies and $6-20 \%$ of all uterine sarcomas. $^{3}$

\section{Correspondence:}

Dr. Poornima Vijayan, $M D$

Department of pathology,

MES Medical College, Perinthalmanna, Kerala, India

E-mail:poornima_vij@yahoo.co.in
Distinguishing LGESS from benign smooth muscle proliferations like cellular leiomyoma can be challenging, but because of differing treatment and prognosis, this distinction is important. Its clinical recognition may be difficult and the diagnosis is often made postoperatively after histopathological examination. Preoperative diagnosis is mostly a uterine fibroid. ${ }^{4}$

Although the presence of an EST is sometimes established by curettage, a definitive diagnosis of LGESS can be made if myometrial and/or vascular invasion is identified in the tissue fragments. Nevertheless, a hysterectomy is usually required to permit the thorough evaluation of the tumor margin which is necessary to distinguish a stromal sarcoma from a benign stromal nodule. ${ }^{5}$

Due to its rarity, ESS has only been reported in a few studies, and these were limited to case reports or retrospective 


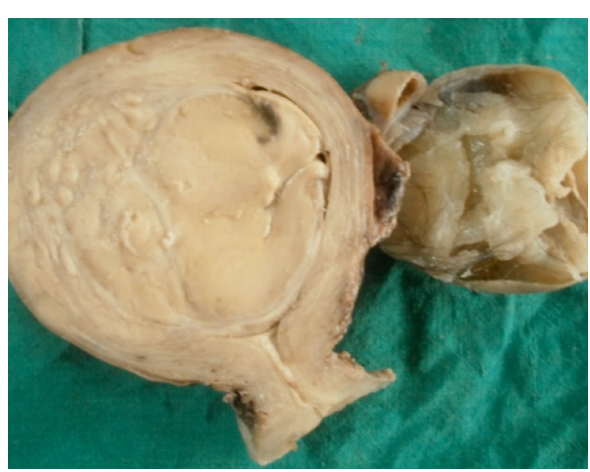

Figure 1: Enlarged uterus with a well delineated predominantly intramural fleshy mass with bulging cut surface. Attached ovary is enlarged showing hair and pultaceous material.

analysis based on a small number of patients. By this study, we have attempted to document the histologic features of LGESS with discussion of the differential diagnoses to contribute to this rare spectrum of uterine neoplasms.

\section{CASE SERIES}

We report a series of 4 cases of low grade endometrial stromal sarcoma reported within a 5 year periodbetween 2010 to 2015 . The salient clinical details of all the cases are highlighted in Table 1.

All four cases were clinically diagnosed as fibroid uterus. In two of the young patients, myomectomy was performed, which was later followed up with total abdominal hysterectomy with bilateral salphingo-oopherectomy after a diagnosis of ESS was made on the myomectomy specimens. The ultrasound examination in all cases were reported as suggestive of large intramural fibroids.

In all four of our cases, the tumor size was large ranging from 15 to $25 \mathrm{~cm}$ in greatest dimension. All were solitary, well delineated, predominantly intramural tumors. The cut surface ranged from pale white to pale yellow, fleshy with bulging cut surface, unlike the classical white firm whorled cut surface of leiomyoma (fig.1). In one case the tumor was seen permeating the myometrium as infiltrating cords.

Microscopically all cases showed a densely cellular tumor composed of uniform oval to spindle cells bearing a close resemblance to normal endometrial stromal cells. Tumor cells were seen arranged around spiral arteriole like vessels. All four cases lacked significant atypia, pleomorphism and mitosis(fig. 2A\&2B). Immunoprofile was similar in all four cases with CD10 (fig. 3A\&3B), and Vimentin (fig. 4A\&4B), showing strong positivity in the tumor cells while, SMA and Desmin showed only focal positivity in areas of smooth muscle differentiation (fig.5A\&5B).

\section{DISCUSSION}

Endometrial stromal tumors are rare uterine malignancies composed of cells resembling those of proliferative endometrial stroma. They are subdivided into benign and malignant groups based on the type of tumor margin. Those with pushing margins are benign stromal nodules, while those with infiltrating margins are stromal sarcomas. Endometrial stromal sarcomas were previously subdivided into low or high grade categories depending upon mitotic index but now the latter are termed poorly differentiated or undifferentiated uterine sarcoma due to its aggressive clinical course. ${ }^{2}$

LGESS usually affects women in the pre and perimenopausal age groups, with a median age being 45 and 57 years.But in our study, two of our cases were young women aged 26 and 32 respectively. Symptoms at presentation include abnormal vaginal bleeding, progressive menorrhagia and abdominal pain as seen in our cases. ${ }^{3}$ Preoperatively they are most commonly misdiagnosed as fibroid uterus and managed accordingly. ${ }^{6}$ The pathogenesis of these lesion remains unknown, but exposures to tamoxifen and unopposed estrogens or prior irradiation have been implicated in some cases. At presentation, extrauterine pelvic extension, most commonly involving the ovary, is found in up to $1 / 3$ of patients.

LGESS show neoplastic stromal cells resembling those of
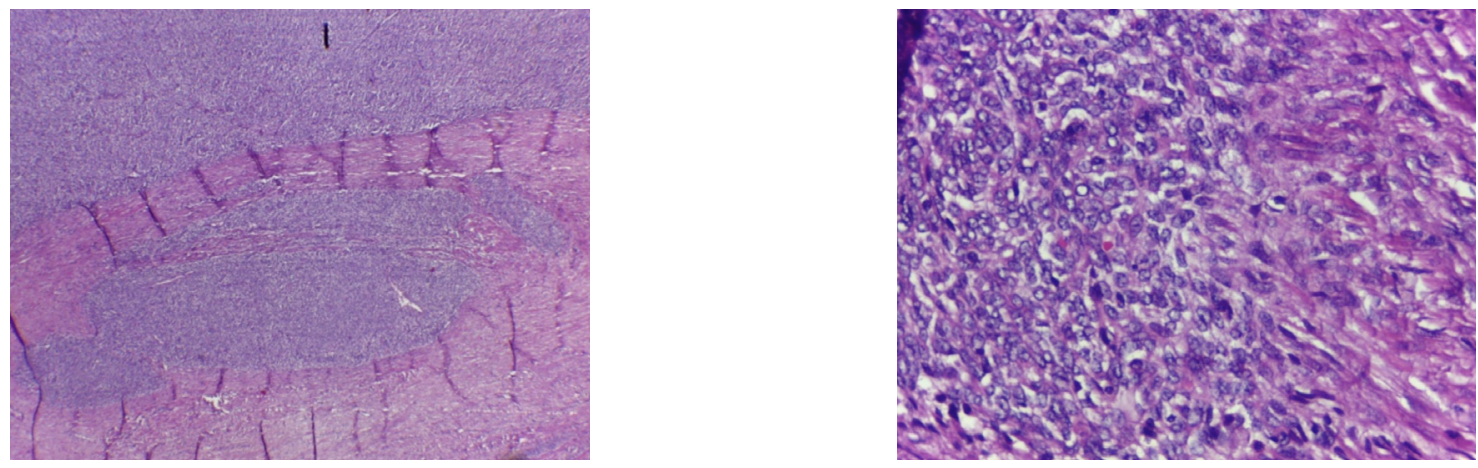

Figure 2A: Tongues of tumor infiltrating the myometrium (HE Stain, X4).

Figure 2B: Uniform oval tumor cells with minimal atypia with foci of smooth muscle differentiation (HE Stain, X20). 


\begin{tabular}{|c|c|c|c|c|c|}
\hline Case No & Age & Signs and symptoms & Clinical findings & Clinical diagnosis & Surgery performed \\
\hline 1. & $26 / f$ & $\begin{array}{l}\text { Abdominal distension, } \\
\text { vague discomfort }\end{array}$ & $\begin{array}{l}\text { Mass per abdomen, } 34 \text { week } \\
\text { sized uterus }\end{array}$ & Fibroid uterus & $\begin{array}{l}\text { Myomectomy, later followed by } \\
\text { TAH+BSO }\end{array}$ \\
\hline 2. & $48 / f$ & $\begin{array}{l}\text { Abdominal pain, disten- } \\
\text { sion and menorrhagia }\end{array}$ & $\begin{array}{l}\text { Mass per abdomen, } 26 \text { week } \\
\text { sized uterus }\end{array}$ & Fibroid uterus & $\mathrm{TAH}+\mathrm{BSO}$ \\
\hline 3. & $32 / f$ & $\begin{array}{l}\text { Vague abdominal dis- } \\
\text { comfort }\end{array}$ & Mass per abdomen & Fibroid uterus & $\begin{array}{l}\text { Myomectomy, later followed by } \\
\text { TAH+BSO }\end{array}$ \\
\hline 4. & $45 / \mathrm{f}$ & $\begin{array}{l}\text { Abdominal distension and } \\
\text { menorrhagia }\end{array}$ & Mass per abdomen & Fibroid uterus & $\mathrm{TAH}+\mathrm{BSO}$ \\
\hline
\end{tabular}

(TAH+BSO - total abdominal hysterectomy with bilateral salphingo-oopherectomy)
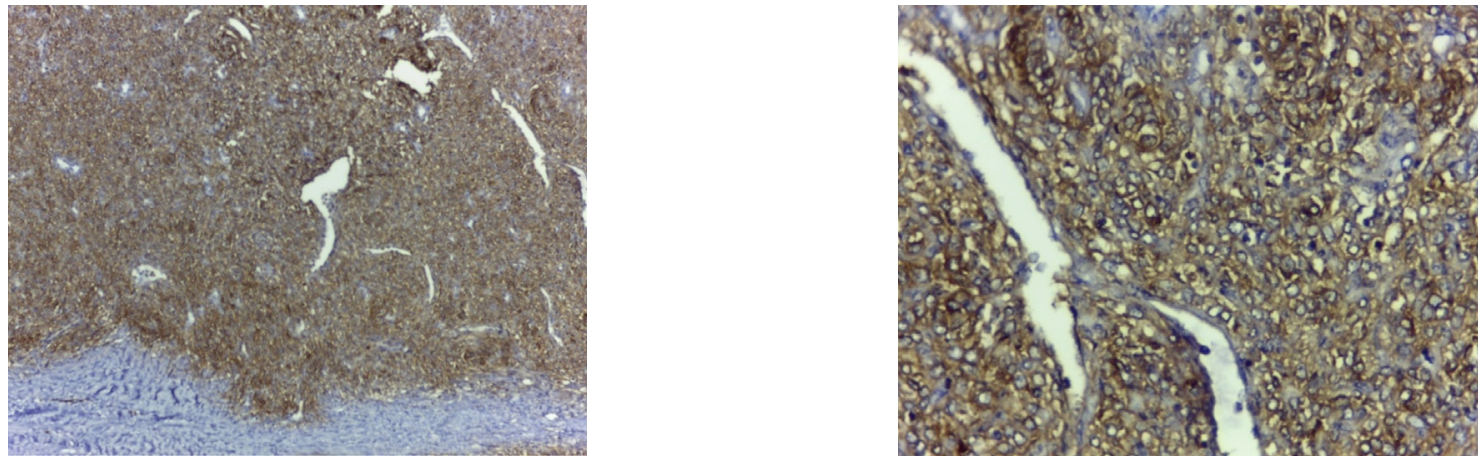

Figure $3 A \& B$ : CD10 strongly positive in tumor cells [IHC, $4 X(2 A), 40 X(2 B)]$.
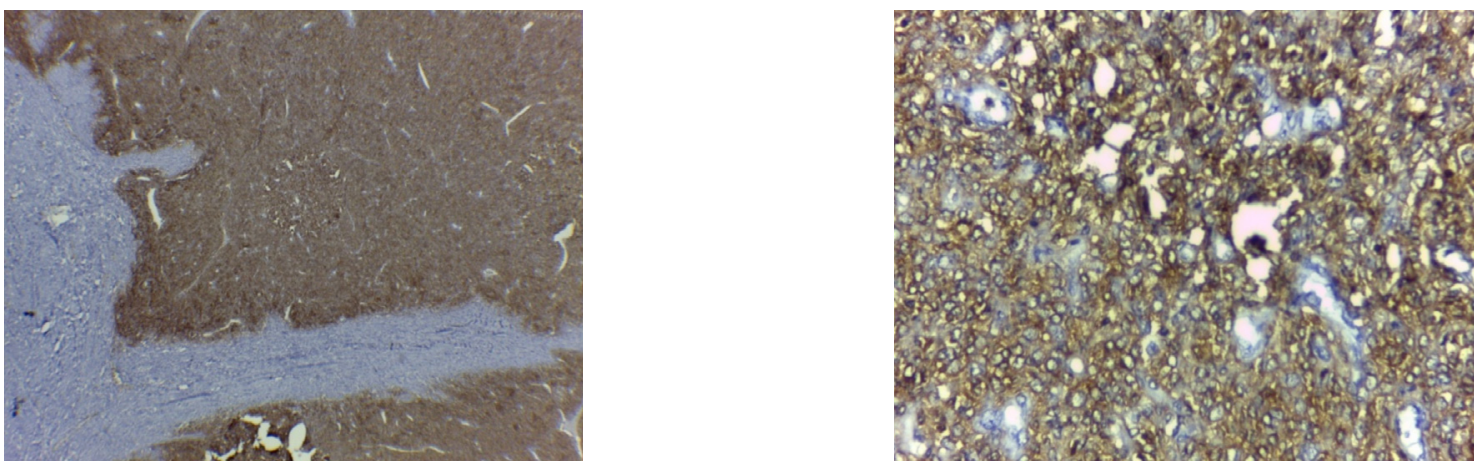

Figure $4 A \& B$ : Vimentin strongly positive in tumor cells [IHC, $4 x(3 A), 40 X(3 B)]$.

proliferative endometrium. The tumor cells are monotonous with uniform size and shape having round to ovoid nuclei with fine granular chromatin, small inconspicuous nucleoli and small amount of amphophilic cytoplasm with indistinct cell borders. Numerous thin walled small arteriolar types of vessels are characteristically present. Reticulin fibres surround individual cells in basket weave pattern. Plaques and zones of hyaline fibrosis are common and aggregates of foam cells or foci of necrosis are occasionally present. Rarely, abundant myxoid stroma widely separates tumor cells. ${ }^{2}$ Atypia, pleomorphism and mitosis are usually infrequent as in our cases.

The differential diagnosis of LGESS includes ESN, cellular leiomyoma, cellular intravenous leiomyomatosis, cellular endometrial polyp and various soft tissue neoplasms. ESN is a tumor composed of cells closely resembling those of endometrial stroma having non-infilterative border with minimal cytologic atypia and without vascular invasion. Hence, extensive sampling of tumor margins and detecting vascular invasion are extremely important in distinguishing between the two. ${ }^{7}$

Distinguishing LGESS from benign smooth muscle proliferations like cellular leiomyoma can be challenging, especially since ESS can show areas of smooth muscle differentiation. Fascicular areas, thick walled blood vessels and cleft-like spaces in cellular leiomyoma, more often seen at the periphery of the tumor are the features which differentiate it with LGESS. In uncertainty, antibodies to smooth muscle myosin-heavy chain, CD10, calponin, smooth muscle actin and desmin can reliably distinguish 

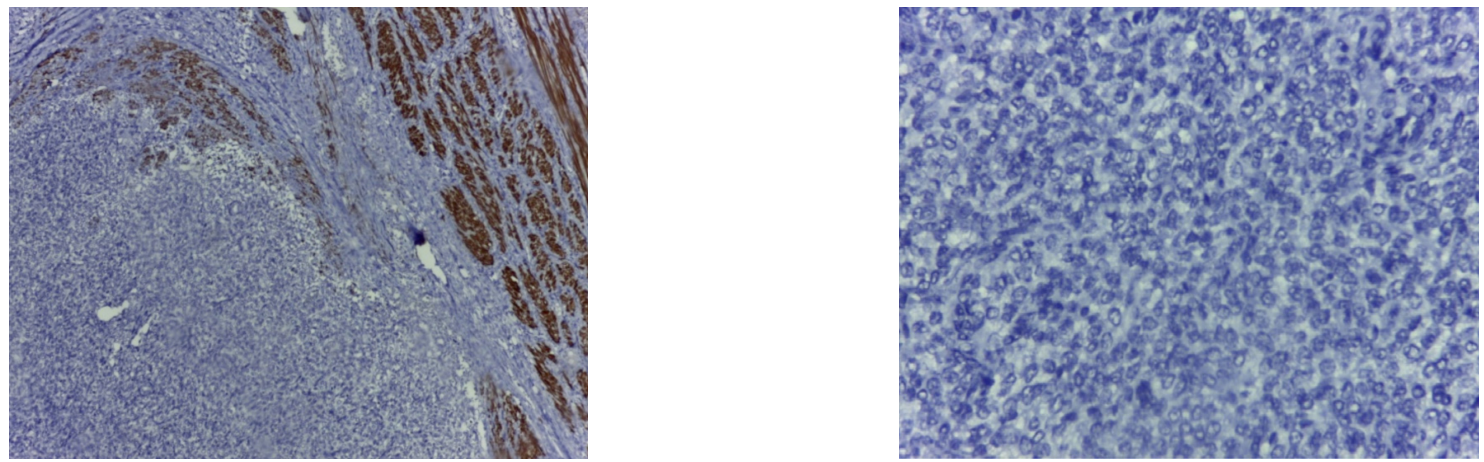

Figure 1: Enlarged uterus with a well delineated predominantly intramural fleshy mass with bulging cut surface. Attached ovary is enlarged showing hair and pultaceous material.

between LGESS and leiomyomas. This correct classification is important due to the differences in clinical behavior and treatment. ${ }^{4}$

ESS may be a diagnostic challenge especially when they are present in an extrauterine site. Owing to the presence of an arborizing vasculature and cells with an undifferentiated appearance, ESS can be confused with several soft tissue neoplasms like hemangiopericytoma, solitary fibrous tumor and synovial sarcoma. Most ESS are CD10 positive combined with antiestrogen receptor and CD $34 .{ }^{8}$

Surgery is fundamental for primary treatment of LGESS consisting of total abdominal hysterectomy with bilateral salpingo-oophorectomy. Regardless of patient's age, preservation of ovarian tissue is not recommended because of likelihood of ovarian metastasis. Because of the high rates of recurrence, patients may receive also adjuvant radiation or hormonal treatment with progestational agents or aromatase inhibitors. ${ }^{4}$

Low grade endometrial stromal sarcomas are indolent tumors with a favorable prognosis. But it is notorious for late recurrences even in patients with stage I disease; thus, long term follow-up is required. About one third of patients develop recurrences, most commonly in the pelvis and abdomen. ${ }^{3}$

Tumor size, mitotic count, tumor stage, histologic grade, involvement of surgical margins by tumor, menopausal status, and age have been reported to have prognostic significance in various studies. ${ }^{3}$ In a study of 113 cases of low-grade endometrial stromal sarcomas by Chang KL et al, mitotic activity and cytological atypia were not found to be predictive of tumor recurrence in stage I tumors, while size correlated poorly with outcome as tumors $<4 \mathrm{~cm}$ in diameter also recurred. For the patients at stage I, overall 5 year survival was reported to be $94.7 \%{ }^{9}$

\section{CONCLUSION}

Because of rarity of this tumor, diagnosis of LGESS is not frequently encountered in gynecological practice and hence awareness is essential. Since ultrasound and MRI studies are inconclusive, frequently it could be mistaken for a fibroid and managed conservatively with myomectomy especially in a younger woman. Hence a high index of suspicion is required to make a correct preoperative diagnosis so that a properly planned management of the patient can be carried out.

\section{REFERENCES}

1. Ramirez NC, Lawrence WD. Endometrial stromal lesions. In:Weitner Cote Suster Weiss. Modern Surgical Pathology.1 sted.Saunders, Philadelphia, London, New York,2003;pp1327-77.

2. Tavassoli FA, Devilee P. World Health OrganizationClassification of Tumors: Pathology and genetics of the breast and female genital organs, IARC Press; Lyon;2003:230-50.

3. NaderehBehtash, SetarehAkhavan, MitraModares Gilani, Azamsadat Mousavi, FatemehGhaemmaghami and MarjanMoradiMazhari. Low Grade Endometrial Stromal Sarcoma of Uterine: Review of 17 Cases. Acta Medica Iranica 2011;49:619-24. Crossref

4. Pinki Pandey, Alok Dixit, AparnaTanwar, Nanak Chand Mahajan. Low Grade Endometrial Stromal Sarcoma: Report of a Rare Uterine Malignancy. Iranian Journal of Pathology 2013;8:64 -70.

5. Yilmaz A, Rush DS, Soslow DA. Endometrial stromal sarcomas with unusual histologic features. Am J Surg Pathol 2002;26:1142-50. Crossref

6. Feeley KM, Burton JL, Wells M. A diagnostic approach to endometrial biopsies: selected topics. Curr Diagn Pathol 2000;6:13-20. Crossref

7. Baker P, Oliva E. Endometrial stromal tumors of the uterus: A practical approach using conventional morphology and ancillary techniques. J Clin Pathol 2007;60:235-43. Crossref

8. Bhargava R, Shia J, Hummer AJ, Thaler HT, Tornos C, Soslow RA. Distinction of endometrial stromal sarcomas from hemangiopericytomatous tumors using a panel of immunohistochemical stains. Mod Pathol 2005;18:40-7. Crossref

9. Sagae S, Yamashita K, Ishioka S, NishiokaY,Terasawa K, Mori M, Yamashiro K, Kanemoto T Kudo R. Preoperative diagnosis and treatment result in 106 patients with uterine sarcoma in Hokkaido,Japan. Oncology 2004;67:33-9. Crossref 